\title{
ON THE CONVERGENCE OF SEQUENCES OF LINEAR OPERATIONS
}

\author{
R. P. BAILEY
}

1. Introduction. Let $S$ be a sequence $\left\{U_{n}(x)\right\}(n=1,2, \cdots)$ of linear operations defined over the elements $\{x\}$ of a Banach space ${ }^{1}$ $E$ with values lying in a second $B$ space $E_{1}$. The fundamental convergence theorem associated with such sequences may be stated as follows:

BANACH-Steinhaus THEOREM. For the convergence of $S$ over $E$ it is necessary and sufficient that $S$ converge over a set $H$ dense in a sphere $K$ of $E$ and that the set of norms $\left\{\left|U_{n}\right|\right\}$ be bounded.

The solution of convergence problems involving sequences of positive functionals defined on certain function-spaces ${ }^{2}$ led the author to investigate a different form of this convergence criterion which seems to have a more direct practical application in some special cases of importance. The development hinges on the fact that from a certain point of view every linear operation has domains over which it is "positive."

2. A new convergence criterion. Let us make the following definition :

Definition. $A$ set of points $P$ in a normed vector space will be called a p-set provided that, for every pair of points $z$ and $z^{\prime}$ of $P$, $\left\|z-z^{\prime}\right\| \leqq\left\|z+z^{\prime}\right\|$.

Such a set has many of the characteristic properties of a set of real numbers all of the same sign. In effect, we have extended the notion of "positiveness" to the elements of an abstract space.

In terms of this definition we may now state the following theorem:

THEOREM 1. For the convergence of $S$ over $E$, it is sufficient that there exist a sphere $K$ of $E$ such that (a) $S$ converges over a set $H$ dense in $K$ and (b) for all fixed $n$ sufficiently large, the set of transforms $\left\{U_{n}(x)\right\}$ $(x \subset K)$, is a p-set, $P_{n}$.

Presented to the Society, February 20, 1937 under the title $A$ note on the convergence of linear operations; received by the editors April 7, 1942.

${ }^{1}$ A space of type B. Cf. Banach, Théorie des opérations linéaires, Warsaw, 1932, p. 53. In general we follow the terminology of this treatise.

${ }^{2}$ R. P. Bailey, Convergence of sequences of positive linear functional operations, Duke Math. J. vol. 2 (1936) pp. 287-303. 
Assuming the conditions of Theorem 1 are satisfied, it is possible to determine a sphere $K^{\prime} \subset K$ of center $\xi \subset H$ and radius $r>0$. Let $\xi^{\prime}=\xi / r$. Then, if $\|x\| \leqq 1$,

$$
\left\|\xi-r\left(\xi^{\prime} \pm x\right)\right\|=\|r x\| \leqq r,
$$

so that $r\left(\xi^{\prime} \pm x\right) \subset K^{\prime} \subset K$. Hence, by our hypothesis, there exists a positive integer $N$, such that, for $n \geqq N$ and $\|x\| \leqq 1$,

$$
P_{n} \supset U_{n}\left[r\left(\xi^{\prime} \pm x\right)\right]=\left[U_{n}\left(r \xi^{\prime}\right) \pm U_{n}(r x)\right] .
$$

Setting $z=U_{n}\left(r \xi^{\prime}\right)+U_{n}(r x)$ and $z^{\prime}=U_{n}\left(r \xi^{\prime}\right)-U_{n}(r x)$, since $\left\|z-z^{\prime}\right\|$ $\leqq\left\|z+z^{\prime}\right\|$, we may conclude

$$
\left\|U_{n}(x)\right\| \leqq\left\|U_{n}\left(\xi^{\prime}\right)\right\|, \quad\|x\| \leqq 1, n \geqq N .
$$

Thus $\left|U_{n}\right| \leqq\left\|U_{n}\left(\xi^{\prime}\right)\right\|(n \geqq N)$.

But the sequence $S$ converges at $\xi$ and hence at $\xi^{\prime}$. It follows that the set $\left\{\left\|U_{n}\left(\xi^{\prime}\right)\right\|\right\}(n=1,2, \cdots)$ is bounded, and therefore the set of norms $\left\{\left|U_{n}\right|\right\}$ is bounded. Thus the Banach-Steinhaus conditions are satisfied, and $S$ converges at all points of $E$. This completes the proof of Theorem 1.

As an illustration consider the sequences of so-called "positive" linear functional operations, that is, linear operations which associate real numbers $U(x)$ with the elements $x=x(t)$ of a function-space in such a way that $U(x) \geqq 0$ whenever $x(t) \geqq 0$. For instance let $\left\{\alpha_{n}(t)\right\}$ $(n=1,2, \cdots)$ be a set of monotone non-decreasing functions defined on $(0,1)$. The functionals $U_{n}(x)=\int_{0}^{1} x(t) d \alpha_{n}(t)(n=1,2, \cdots)$ are positive linear functionals over the $B$ space $C$ consisting of the continuous functions $\{x(t)\}$ defined on $(0,1)$ with norm $\|x\|=\max _{0 \leqq t \leqq 1}|x(t)|$, and therefore obviously transform spheres of non-negative functions into the $p$-set consisting of the positive real numbers. It follows that the sequence $\left\{U_{n}(x)\right\}$ cannot converge over a set dense in a sphere of $C$ without converging over the whole of $C$.

It should not be assumed, however, that the only practical value of the criterion lies in its application to sequences of positive functionals. All of the better known Banach spaces contain easily recognized $p$-sets of a very extensive character. We may point, for instance, to the set of non-negative functions of $C$ space, the set of points $x=\left(x_{1}, x_{2}, \cdots, x_{n}\right)$ of Euclidean $n$-space such that $x_{i} \geqq 0$ $(i=1,2, \cdots, n)$, and the set of functions $\{x(t)\}$ Lebesgue square integrable on $(0,1)$ with the norm $\|x\|=\left[\int_{0}^{1} x^{2} d t\right]^{1 / 2}$ which consists of those functions having non-negative Fourier coefficients. In fact, we shall prove in what follows that the sphere with arbitrary center $z_{0}$ and radius $\left\|z_{0}\right\| / 2$ in any $B$ anach space is a $p$-set. 
In other words, the real value of the criterion lies in the fact that it is frequently obvious at a glance that certain spheres in $E$ transform into $p$-sets in $E_{1}$, while the boundedness of the norms may be difficult to establish.

3. Necessity of the criterion. In an abstract of this paper ${ }^{3}$ the author made an error in stating that the conditions of Theorem 1 were necessary for convergence, as well as sufficient. The question of necessity is clarified in this section. We shall assume the Banach-Steinhaus conditions are satisfied, and ask under what circumstances it is necessary that some sphere of $E$ transform into $p$-sets in $E_{1}$.

By hypothesis there exists a number $M>0$ such that $\left|U_{n}\right| \leqq M$ for all $n$, and $S$ converges at all points of $E$ to a linear operation $U(x)$. It is convenient to divide the discussion into two parts, depending upon whether or not $U(x)$ vanishes identically over $E$. Denote by $\theta_{1}$ the zero element of $E_{1}$.

Case 1. $\left(U(x) \not \equiv \theta_{1}\right.$.) Choose $\xi \subset E$ such that $U(\xi) \neq \theta_{1}$. Since $\|U(\xi)\|>0$, positive numbers $\epsilon>0, r>0$ may be chosen such that $\epsilon+r M \leqq\|U(\xi)\| / 2$. If $x$ is any point of the sphere $K$ defined by the condition $\|\xi-x\| \leqq r$, and $N$ be so chosen that $\left\|U(\xi)-U_{n}(\xi)\right\| \leqq \epsilon$ $(n \geqq N)$, it follows that for $n \geqq N$ and $\|\xi-x\| \leqq r$,

$$
\begin{aligned}
\left\|U(\xi)-U_{n}(x)\right\| & \leqq\left\|U(\xi)-U_{n}(\xi)\right\|+\left\|U_{n}(\xi-x)\right\| \\
& \leqq \epsilon+r M \\
& \leqq\|U(\xi)\| / 2 .
\end{aligned}
$$

That is, for $n \geqq N$, and $\|\xi-x\| \leqq r$, all transforms $\left\{z=U_{n}(x)\right\}$ lie in a sphere of $E_{1}$ defined by the condition

$$
\|U(\xi)-z\| \leqq\|U(\xi)\| / 2 \text {. }
$$

Moreover this spherical set is a $p$-set, for if $z$ and $z^{\prime}$ are any two of its points,

$$
\begin{aligned}
\left\|z-z^{\prime}\right\| & \leqq\|z-U(\xi)\|+\left\|U(\xi)-z^{\prime}\right\| \\
& \leqq\|U(\xi)\|
\end{aligned}
$$

while

$$
\begin{aligned}
\left\|z+z^{\prime}\right\| & =\left\|z-U(\xi)+z^{\prime}-U(\xi)+2 U(\xi)\right\| \\
& \geqq 2\|U(\xi)\|-\|z-U(\xi)\|-\left\|z^{\prime}-U(\xi)\right\| \\
& \geqq 2\|U(\xi)\|-\|U(\xi)\| \\
& \geqq\|U(\xi)\| .
\end{aligned}
$$

${ }^{3}$ Bull. Amer. Math. Soc. abstract 43-3-97. 
That is, the criterion of Theorem 1 is satisfied. We have proved this theorem:

THEOREM 2. The conditions of Theorem 1 are necessary for the convergence of $S$ over $E$ to a linear operation $U(x)$ which does not vanish identically.

Case 2. $\left(U(x) \equiv \theta_{1}\right.$.) An example will be furnished to show that no necessity exists in this case. Let $E$ consist of the set of continuous functions $\{x(t)\}$ defined on $(0,1)$ with $x(0)=0$. We use the customary norm $\|x\|=\max _{0 \leqq t \leqq 1}|x(t)|$. If $U_{n}(x)=n \int_{0}^{1 / n} x(t) d t \quad(n=1,2, \cdots)$, clearly $\lim _{n \rightarrow \infty} U_{n}(x)=0$ at all points of $E$. Nevertheless, it is impossible to choose $N>0$, and a sphere $K$ of $E$, such that for fixed $n \geqq N$ and $x \subset K$, the transforms $\left\{U_{n}(x)\right\}$ constitute $p$-sets $P_{n}$. For, suppose $K$ be any arbitrary sphere $\left\|x_{0}-x\right\| \leqq r$ with center $x_{0}$. Since $x_{0}(0)=0$, it is possible to determine a point $t_{0}\left(0<t_{0}<1\right)$ such that $\max \left|x_{0}(t)\right|$ $\left(0 \leqq t \leqq t_{0}\right)$ is arbitrarily small. Clearly, if $t_{0}$ is sufficiently small, there exist functions $x_{1}(t)$ and $x_{2}(t)$ having the following properties:

$$
x_{1}(t)>0, \quad x_{2}(t)<0, \quad 0<t \leqq t_{0},
$$

But now, if $n \geqq 1 / t_{0}, U_{n}\left(x_{1}\right)>0$, while $U_{n}\left(x_{2}\right)<0$. The set of transforms $\left\{U_{n}(x)\right\}(x \subset K)$ therefore cannot form a $p$-set, no matter what sphere $K$ is selected, if $n$ is sufficiently large.

4. Convergence on a locally compact $B$ space. We have shown in the preceding section that the conditions of Theorem 1 need not be satisfied in all cases to obtain convergence. However, it is possible to establish the following criterion:

THEOREM 3. For the convergence of $S$ over a $B$ space $E$ which is locally compact, the conditions of Theorem 1 are necessary and sufficient. ${ }^{4}$

We have only to demonstrate necessity in the case $U(x) \equiv \theta_{1}$. The remainder follows at once from Theorems 1 and 2 . We shall need the following lemma.

Lemma. A sequence $\left\{F_{n}(x)\right\}(n=1,2, \cdots)$ of linear operations defined over a locally compact $B$ space $E$ may not possess both of the following characteristics:

$$
\begin{array}{rr}
\left|F_{n}\right|=1, & n=1,2, \cdots, \\
\liminf _{n \rightarrow \infty}\left\|F_{n}(x)\right\|=0 \quad \text { for all } x \text { of } E .^{5}
\end{array}
$$

${ }^{4}$ We may mention in passing that the property of local compactness holds if, and only if, the space has a finite number of dimensions.

${ }^{5}$ Note that the operations $U_{n}(x) \equiv n \int_{0}^{1 / n} x(t) d t$ defined in $\$ 3$ do possess both properties. 
Suppose $\left\{G_{n}(x)\right\}(n=1,2, \cdots)$ is any infinite selection of the operations $\left\{F_{n}(x)\right\}$. Let $\epsilon>0$ be any arbitrarily specified real positive number. For each $n=1,2, \cdots$ there exists a point $\xi_{n} \subset E$ such that $\left\|\xi_{n}\right\| \leqq 1$ and $\left\|G_{n}\left(\xi_{n}\right)\right\| \geqq 1-\epsilon$. Since the unit sphere is compact, it is possible to extract from the sequence $\left\{\xi_{n}\right\}$ a subsequence $\left\{\xi_{n_{k}}\right\}$ which converges to a point $\xi$ of $E$. For $n_{k}$ sufficiently large, $\left\|\xi-\xi_{n_{k}}\right\| \leqq \epsilon$. Hence, for all $n_{k}$ sufficiently large,

$$
\begin{aligned}
\left\|G_{n_{k}}(\xi)\right\| & =\left\|G_{n_{k}}\left(\xi_{n_{k}}\right)+G_{n_{k}}\left(\xi-\xi_{n_{k}}\right)\right\| \\
& \geqq\left\|G_{n_{k}}\left(\xi_{n_{k}}\right)\right\|-\left\|G_{n_{k}}\left(\xi-\xi_{n_{k}}\right)\right\| \\
& \geqq 1-2 \epsilon,
\end{aligned}
$$

and therefore $\lim \sup _{n \rightarrow \infty}\left\|G_{n}(\xi)\right\| \geqq 1$. Since $\left\{G_{n}(x)\right\}$ is any arbitrary infinite selection of the operations $\left\{F_{n}(x)\right\}$, this contradicts (ii).

Now consider any arbitrary sequence $\left\{U_{n}(x)\right\}$ defined over a locally compact space $E$, such that $\lim _{n \rightarrow \infty} U_{n}(x)=U(x)$ vanishes at all points of $E$. We wish to show that there exists a sphere $K$ of $E$ such that $\left\{U_{n}(x)\right\}(x \subset K)$ constitutes a $p$-set $P_{n}$, for all $n$ sufficiently large.

The contradomain of an operation $U_{n}(x) \equiv \theta_{1}(x \subset E)$ is obviously a $p$-set. Hence consider only the subsequence $\left\{U_{n_{k}}(x)\right\}$ of $\left\{U_{n}(x)\right\}$ consisting of those operations which do not vanish identically over ${ }^{6} E$. Since $\left|U_{n_{k}}\right|>0$, we may set

$$
F_{n_{k}}(x)=\left|U_{n_{k}}\right|^{-1} \cdot U_{n_{k}}(x), \quad x \subset E .
$$

Since $\left|F_{n_{k}}\right|=1(k=1,2, \cdots)$, it is impossible that $\lim _{\inf _{k \rightarrow \infty}}\left\|F_{n_{k}}(x)\right\|$ $=0$ at all points of $E$, by the previously proved lemma. Hence there exists a point $\xi \neq \theta$, and a number $r>0$, such that

$$
\liminf _{k \rightarrow \infty}\left|U_{n_{k}}\right|^{-1} \cdot\left\|U_{n_{k}}(\xi)\right\|>2 r>0 .
$$

Therefore it is possible to fix $N$ so large, that for $n_{k}>N$,

or

$$
\left|U_{n_{k}}\right|^{-1} \cdot|| U_{n_{k}}(\xi)|| \geqq 2 r,
$$

$$
r\left|U_{n_{k}}\right| \leqq\left\|U_{n_{k}}(\xi)\right\| / 2 \text {. }
$$

Now, if $\|x-\xi\| \leqq r$, and $n_{k}>N$,

$$
\left\|U_{n_{k}}(x)-U_{n_{k}}(\xi)\right\| \leqq r\left|U_{n_{k}}\right| \leqq\left\|U_{n_{k}}(\xi)\right\| / 2 .
$$

${ }^{6} \mathrm{We}$ assume the subsequence is infinite; in the contrary case nothing remains to be proved. 
Since $\left\|U_{n_{k}}(\xi)\right\|>0$, the sphere $\left\|z-U_{n_{k}}(\xi)\right\| \leqq\left\|U_{n_{k}}(\xi)\right\| / 2$, is nonvacuous. That such a sphere is a $p$-set was demonstrated in $\$ 3$. The sphere $K$ we were required to construct has therefore been shown to exist, and Theorem 3 is proved.

Lafayette College

\title{
ON THE APPROXIMATION OF FUNCTIONS BY SUMS OF ORTHONORMAL FUNCTIONS
}

\author{
EDWIN N. OBERG
}

1. Introduction. The main object of this paper is to derive, in a simple manner, upper bounds for the norms of the derivative of

$$
\sum_{i=0}^{n} a_{i} \phi_{i}(x)
$$

in $C$ and $L^{2}$ spaces, where the $a_{i}$ are arbitrary constants, and $\left\{\phi_{i}(x)\right\}$ is any set of functions on a given finite or infinite interval $(a, b)$. We apply our method, properly modified, first to the case where the $\phi_{i}(x)$ are characteristic solutions of conjugate sets of integral equations, then to other classes of functions whose first derivatives $\left\{\phi_{i}^{\prime}(x)\right\}$ are orthogonal with respect to a weight function $\sigma(x)$. Finally, we apply our results to the question of convergence of sums ${ }^{1}$ of type (1) that minimize

$$
\int_{a}^{b} \rho(x)\left|f(x)-\sum_{i=0}^{n} a_{i} \phi_{i}(x)\right|^{m} d x, \quad m>0 .
$$

The leading results of our investigation may be summarized briefly as follows:

$$
\left|\frac{d}{d x} \sum_{i=0}^{n} a_{i} \phi_{i}(x)\right| \leqq \lambda_{n} k(x)\left(\int_{a}^{b}\left[\sum_{i=0}^{n} a_{i} \phi_{i}(x)\right]^{2} d x\right)^{1 / 2}
$$

where $\lambda_{n}$ is a positive number that increases with $n$ and $k(x)$ is a func-

Presented to the Society, September 5, 1941 under the title Notes on the approximation of functions by sums of orthonormal functions; received by the editors February 2, 1942.

${ }^{1}$ For the specialized cases when the approximating functions are trigonometric sums or polynomials, see D. Jackson, The theory of approximation, Amer. Math. Soc. Colloquium Publications vol. 11, 1930, pp. 86-89, 96-101. 